Faculdade

de Ciências Econômicas UFRGS

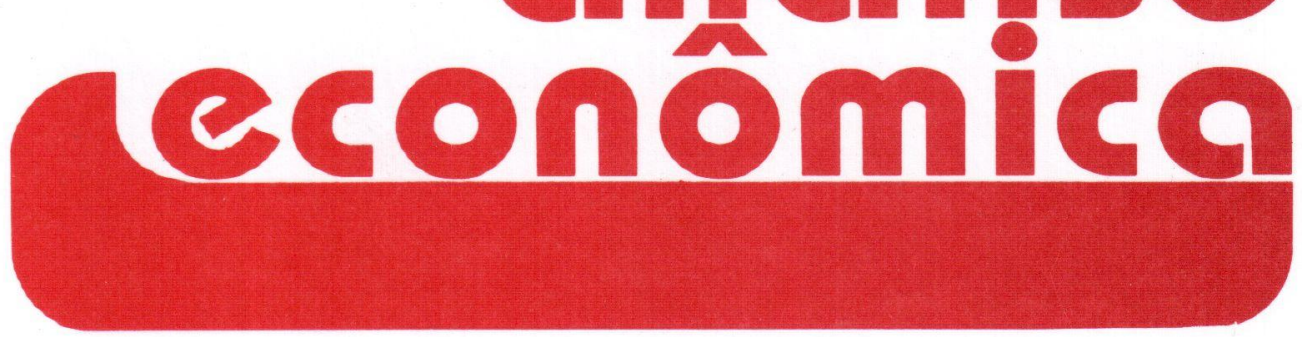

- UM SÉCULOdE INFLAÇÃO: A EVIDÊNCIA EMPÍRICA Fábio Sá Earp

- REGIÕES-CHAVENAINTEGRAÇÃo ECONÔMICA REGIONAL Nali de Jesus de Souza

- THESRAFFIAN INTERPRETATION OF RICARDO: ACRITIQUE José Ricardo Bezerra Nogueira

- QUALIDADE DO MEIO AMBIENTE EFALHAS DE MERCADO

Francisco de Sousa Ramos

- EFEITOS ECONÔMICOS DEESTRATÉGIAS DE PROMOÇÃODE EXPORTAÇÕES Wilson da Cruz Vieira

- "KEYNESIANS", MONETARISTS,NEW CLASSICALS AND NEW KEYNESIANS Fernando Ferrari Filho

- DISTRIBUIÇÃO DA RENDA DO TRABALHO NAINDÚSTRIA BRASILEIRA

Antonio Lisboa Teles da Rosa

- CURSO dE EXTENSÃO EM ECONOMIA PREPARATÓRIO PARA A ANPEC

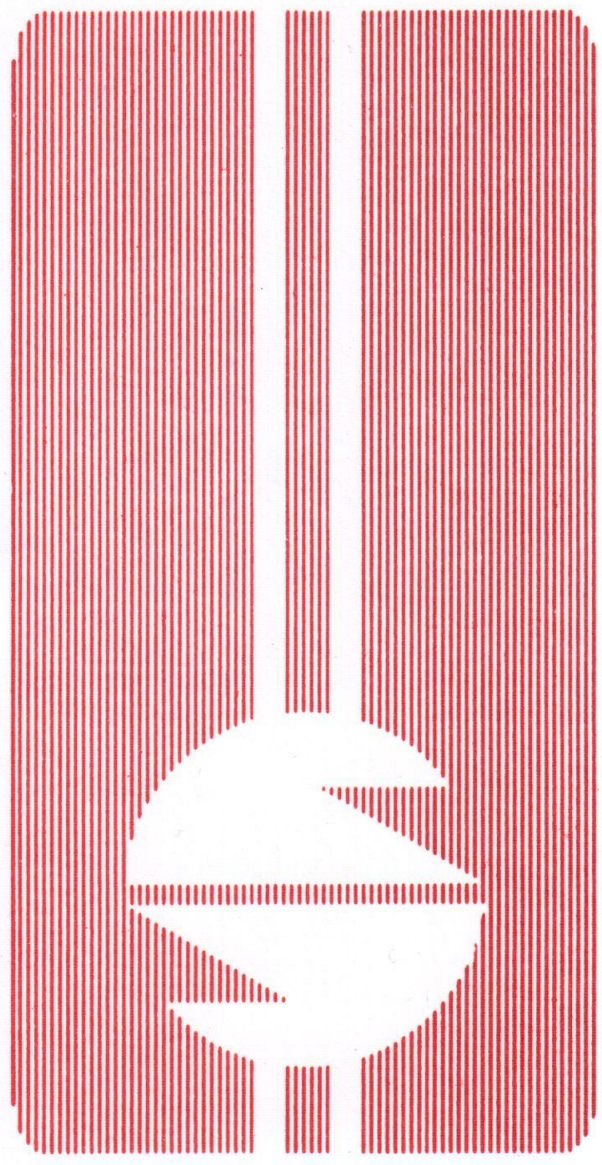


UNIVERSIDADE FEDERAL DO RIO GRANDE DO SUL

Reitor. Prof. Hélgio Henrique Casses Trindade

FACULDADE DE CIÊNCIAS ECONÔMICAS

Diretor. Prof. Pedro Cézar Dutra Fonseca

CENTRO DE ESTUDOS E PEQUISAS ECONÔMICAS

Diretor. Prof. Paulo Alexandre Spohr

DEPARTAMENTO DE CIÊNCIAS ECONÔMICAS

Chefe: Prof. Gentil Corazza

CURSO DE PÓS-GRADUAÇĀO EM ECONOMIA

Coordenador Prof Roberto Camps Moraes

CURSO DE PÓS-GRADUAÇÃO EM ECONOMIA RURAL

Coordenador. Prof. Aray Miguel Feldens

CONSELHO EDITORIAL: Achyles Barcelos da Costa, Aray Miguel Feldens, Atos Freitas Grawunder, Carlos Augusto Crusius, Eugenio Lagemann, Fernando Ferrari Filho, Gentil Corazza, Marcelo Savino Portugal, Maria Imilda da Costa e Silva, Nali de Jesus de Souza, Nuno R. L. de Figueiredo Pinto, Otília Beatriz K Carrion, Paulo Alexandre Spohr, Paulo Dabdab Waquil, Pedro Cezar Dutra Fonseca, Roberto Camps Moraes, Valter José Stulp, David Garlow (Wharton Econometrics Forecasts Association, E.U.A.), Edgar Augusto Lanzer (UFSC), Eleutério F. S. Prado (USP), Fernando de Holanda Barbosa (FGV/RJ), Gustavo Franco (PUC/RJ), JJoão Rogério Sanson (UFSC), Joaquim Pinto de Andrade (UnB), Juan H. Moldau (USP), Werner Baer (Univ de lllinois, E. U.A.).

COMISSÃO EDITORIAL: Fernando Ferrari Filho, Gentil Corazza, Paulo Dabdab Waquil, Marcelo Savino Portugal, Roberto Camps Moraes.

EDITOR: Nali de Jesus de Souza

SECRETARIA Cláudia Porto Silveira, Jeferson Luis Bittencourt e Vanete Ricachescki (revisão de textos).

FUNDADOR: Prof. Antônio Carlos Santos Rosa

Os materiais publicados na revista Análise Econômica são da exclusiva responsabilidade dos autores. É permitida a reprodução total ou parcial dos trabalhos, desde que seja citada a fonte.

Aceita-se permuta com revistas congêneres. Aceitam-se, também, livros para divulgação, elaboração de resenhas e recensões.

Toda correspondência, material para publicação (vide normas na terceira capa), assinaturas e permutas devem ser dirigidos ao seguinte destinatário:

PROF. NALI DE JESUS DE SOUZA

Revista Análise Econômica

Av. João Pessoa, 52

CEP 90040-000 PORTO ALEGRE - RS, BRASIL

Telefones: (051) 316-3348 e 316-3440

Fax: (051) 225-1067 


\section{REGIÕES-CHAVE NA INTEGRAÇÃO ECONÔMICA REGIONAL}

Nali de Jesus de Souza*

\section{SINOPSE}

Este artigo estabelece um modelo de integração espacial entre diferentes regiöes, a partir de uma matriz de relações comerciais inter-regionais. O modelo fornece indicadores de integraçäo espacial, a mensuração dos efeitos de encadeamento inter-regionais e a definiçäo de regiöes-chave no encadeamento do crescimento no espaço, permitindo maximizar a integraçáo espacial no interior de um mesmo pais, ou no contexto de zonas de integraçäo, como Mercosul ou o NAFTA

\section{CONCEITO DE INTEGRAÇÃO}

Observando um quadro de insumo-produto, constata-se que o sistema econômico desenvolvido apresenta seus diferentes setores fortemente integrados entre si Pelo contrário, a economia subdesenvolvida mostra-se desarticulada no que diz respeito aos seus diferentes segmentos, predominando o isolamento, a dependência e a assimetria. À economia subdesenvolvida faltam partes, não constituindo um todo conectado por relações técnicas (Leontief, 1966, cap.4).

Consequientemente, o desenvolvimento de uma economia pode ser definido como o preenchimento de sua matriz de insumo-produto, através da implantação de novos blocos de atividades, interligando setores até então desconectados, como indústrias vinculadas à agricultura e aos serviços (Bernis, 1966).

O circuito clássico mercado-empresas-famílias pressupõe que todos os agentes interagem de forma integrada, contínua, sem qualquer ruptura. A introdução de novos setores no modelo, como o setor financeiro, o governo e o setor externo, não modifica a hipótese da fluidez dos circuitos entre os diferentes segmentos da economia Considera-se que todos os setores sejam interligados por redes desenvolvidas de transportes e comunicações, favorecendo a circulação de bens e serviços, de pessoas e informações. Os circuitos de propagação dos efeitos de encadeamento estão presentes tanto setorial como espacialmente.

Contudo, na prática, principalmente nos países subdesenvolvidos, não existe integração perfeita entre setores e regiões. Segmentos importantes da agricultura são de subsistência e não passam pelo mercado. Alguns setores ligam-se diretamente com o exterior, com articulações muito fracas com a economia local, pela ausência de atividades de articulação interna. A integração de dois subconjuntos, setores ou regiões, consiste na ampliação do número de relações econômicas entre ambos, em termos de fatores de produção, produtos e informações, de sorte a torná-los um todo mais homogêneo. Com a integração, diferentes subconjuntos tornam-se mais compativeis e suscetíveis de se desenvolverem cada vez mais em direção a um ótimo global (Erbes, 1966, p.7)

\footnotetext{
"Professor Titular do Departamento de Economia e da Pós-Graduação em Economia da UFRGS
}

\begin{tabular}{|c|l|}
\hline $\begin{array}{c}\text { Cód. } A E A \\
946\end{array}$ & $\begin{array}{l}\text { Palavras-chave: Regióes-chave, integração regional, desenvolvimento } \\
\text { econômico }\end{array}$ \\
\hline
\end{tabular}

\begin{tabular}{|l|l|l|l|}
\hline ANÁLISE ECONÔMICA & ANO 14 & Março/ Setembro 96 & p. 16-24 \\
\hline
\end{tabular}


No campo da economia internacional, a integração econômica pode revestir várias formas zonas de livre comércio entre países, união aduaneira, mercado comum e união econômica total (Balassa, 1961, p 13). O conceito que está sendo adotado neste artigo é mais geral, compreendendo a articulação de regiões dentro ou fora de um país, assim como as partes de um conjunto de indústrias no interior ou fora de complexos industriais. Ele compreende, portanto, a integração intersetorial e a integração espacial.

A integração intersetorial ocorre vertical e horizontalmente. Dois setores apresentam-se integrados verticalmente, quando suas relações ocorrem para trás no processo produtivo (backward effects), e mostram-se integrados horizontalmente, quando tais ligaçöes se processam para a frente no referido processo (forward effects) O aumento da produção de uma atividade local, integrada, incrementará direta e indiretamente a produção das atividades ligadas. Quanto maior o grau de integração do parque produtivo local, maiores serão os efeitos de encadeamento de atividades-chave sobre atividades ligadas tecnologicamente.

A expansão da rede industrial local ocorre com a diversificação do parque produtivo, através de atividades que se ligam tecnologicamente e via demanda final com outras atividades da área. Essa diversificação e o aumento do grau de integração intersetorial desenrola-se de três maneiras principais: a) pela implantação de pontos de articulação; b) pela redução do grau de vuinerabilidade dos complexos, e c) pela extensão simples das redes de atividades (Souza, 1978).

Um setor constitui um ponto de articulação quando religa dois conjuntos de atividades até então isolados um do outro, ele serve de lugar de passagem dos efeitos de encadeamento, provenientes de qualquer uma das atividades de cada subconjunto. Um exemplo pode ser fornecido pela introdução de uma fábrica de conservas alimentícias, em determinada região, que passa a consumir chapas metálicas e potes de vidro para embalagens de produtos agrícolas. Essa nova fábrica transmitirá os efeitos de encadeamento dos setores siderúrgico e de fabricação de artigos de vidro para o setor agricola e vice-versa (Souza, 1988).

A redução da vulnerabilidade de um complexo significa a implantação ou o desenvolvimento de ramos capazes de funcionar como pontos de articulação adicionais Quando o complexo possuir apenas um ponto de articulação ele será vulnerável, porque a redução da produção ou o desaparecimento do ponto de articulação existente poderá colocar em depressão todo o complexo. As crises no interior do complexo podem ser ocasionadas, principalmente, se o ponto de articulação for muito sensivel às flutuações conjunturais externas, transportando-as, dessa forma, para o interior do complexo No exempio anterior, uma segunda fábrica consumidora de produtos siderúrgicos, de minerais não-metálicos e de produtos agricolas reduziria a vulnerabilidade de economia local, por constituir um ponto de articulação adicional entre dois extremos da cadeia produtiva

A extensão das redes compreende a implantação de pontos de articulação adicionais e a introdução de qualquer atividade que amplie a dimensão dos complexos, sem serem necessariamente atividades "ponte" ou de interligação Essas novas ligações permitem a retenção dos efeitos de encadeamento das atividades na própria região, promovendo o crescimento das atividades satélites, ligadas às atividades-chave, como setores mais tradicionais, a agricultura e os serviços. As unidades produtivas, até então isoladas, podem ligar-se com o conjunto 
da economia, ampliar sua escala de produção e reduzir seus custos médios $\mathrm{O}$ aumento das interdependências de uma economia eleva a dimensão do mercado interno, fato que favorece a expansão dos investimentos.

A criação de uma fila de atividades interdependentes, em áreas menos desenvolvidas, torna-se possível, inicialmente, pela implantação de complexos de atividades ligadas à agricultura e às atividades extrativas. A criação de mercado interno e o desenvolvimento dos meios de transporte e de comunicações permitem a implantação de outras atividades vinculadas também às exportações. $A$ integração interna de cada área, aumentando a circulação de bens no espaço, proporcionará a integração espacial entre as diferentes regiōes de um mesmo país.

\section{MENSURAÇÃO DA INTEGRAÇÃO ESPACIAL}

Modelo semelhante ao desenvolvido nesta seção foi estabelecido por Metzler (1950), com o objetivo de avaliar os efeitos de investimentos em um sistema de regiões. Um primeiro esboço deste modelo foi realizado por Souza (1977 e 1978).

O grau de integração espacial de uma economia pode ser mensurado a partir de um quadro de comércio inter-regional entre $n$ regiōes (Quadro 1). $O$ elemento $M_{i j}$ do referido quadro indica as importações da região $j$ provenientes da regiäo $i$, alternativamente, representa as exportações da região i para a região $j$. Na diagonal principal tem-se a parte do produto de cada região absorvida pelo mercado local $\left(N_{i}=M_{i j}\right.$, sendo $\left.i=j\right)$ A soma dos elementos de cada linha, englobando a absorção local $\left(N_{i}\right)$, as vendas para o mercado nacional $\left(X_{i N}\right)$ e as exportações para o mercado externo $\left(\mathrm{X}_{\mathrm{i}}\right)$, constituem a produção total de cada região $\left(\mathrm{Y}_{\mathrm{i}}\right)$, como segue:

$$
Y_{i}=N_{i}+X_{i N}+X_{i E}
$$

QUADRO 1 - MATRIZ DOS FLUXOS INTER-REGIONAIS DE BENS E SERVICQOS

\begin{tabular}{|c|c|c|c|}
\hline $\begin{array}{c}\backslash \text { Destino } \\
\text { Origem }\end{array}$ & Região 1 & Região 2 & Região 3 \\
\hline Região 1 & $N_{1}$ & $M_{12}$ & $M_{13}$ \\
\hline Região 2 & $M_{21}$ & $N_{2}$ & $M_{23}$ \\
\hline Região 3 & $M_{31}$ & $M_{32}$ & $N_{3}$ \\
\hline
\end{tabular}

\begin{tabular}{|c|c|c|c|}
\hline$X_{i N}$ & $X_{i E}$ & $X_{i}$ & $Y_{i}$ \\
\hline$X_{1 N}$ & $X_{1 E}$ & $X_{1}$ & $Y_{1}$ \\
\hline$X_{2 N}$ & $X_{2 E}$ & $X_{2}$ & $Y_{2}$ \\
\hline$X_{3 N}$ & $X_{3 E}$ & $X_{3}$ & $Y_{3}$ \\
\hline
\end{tabular}

\begin{tabular}{|l|c|c|c|}
\hline$M_{\mathrm{Ni}}$ & $M_{\mathrm{N} 1}$ & $M_{\mathrm{N} 2}$ & $M_{\mathrm{N} 3}$ \\
\hline$M_{E_{1}}$ & $M_{E_{1}}$ & $M_{E_{2}}$ & $M_{E_{3}}$ \\
\hline$M_{1}$ & $M_{1}$ & $M_{2}$ & $M_{3}$ \\
\hline$D_{1}$ & $D_{1}$ & $D_{2}$ & $D_{3}$ \\
\hline$\left(X_{i}-M_{i}\right)$ & $S_{1}$ & $S_{2}$ & $S_{3}$ \\
\hline$Y_{i}$ & $Y_{1}$ & $Y_{2}$ & $Y_{3}$ \\
\hline
\end{tabular}

sendo as exportações totais da região $i\left(X_{i}\right)$ iguais a $\left(X_{i N}+X_{i E}\right)$ e as vendas para o mercado nacional $\left(X_{i N}\right)$ iguais a $\left(M_{i 1}+M_{i 2}+M_{i 3}+\ldots+M_{i n}\right.$, para $i$ diferente de $\left.j\right)$. A soma dos valores de cada coluna também fornece a produção total $\left(Y_{j}\right)$, igual a despesa total da região $j\left(D_{j}\right)$, mais o saldo de sua balança comercial $\left(S_{j}=X_{j}-M_{j}\right)$ :

$$
Y_{j}=D_{j}+S_{j}
$$

Se o saldo da balança comercial for nulo, a despesa será igual à produção total; se for negativo, a região gasta mais do que produz. A despesa total $\left(D_{j}\right)$ eqüivale à soma das compras que a região $j$ realiza de si própria $\left(N_{j}=M_{i j}\right.$, sendo $\left.i=j\right)$, mais as 
importações que efetua das demais regiões do país $\left(M_{N j}=M_{1 j}+M_{2 j}+\ldots+M_{N j}\right.$, para $i$ diferente de $j$ ), além das compras efetuadas do exterior $\left(M_{e j}\right)$, ou seja:

$$
D_{j}=N_{j}+M_{N j}+M_{E j}
$$

sendo $\left(M_{N j}+M_{E j}\right)$ as importações totais da região $j\left(M_{j}\right)$.

Com $N_{j}=M_{j}$ (para $i=j$ ), a relação (2) pode ser rescrita como:

$$
Y_{j}=M_{1 j}+M_{2 j}+M_{3 j}+\ldots+M_{E j}+S_{j}
$$

Inspirando-se no modelo da base exportadora, ou base econômica (Souza, 1976 ou 1980), pode-se estabelecer os indicadores de integração espacial mostrados no Quadro 2. Supondo que as atividades do mercado interno, as importações, a despesa total e o saldo da balança comercial sejam proporcionais à produção total $\left(Y_{j}\right)$, a partir de (1) obtém-se a seguinte relação

$$
1=b_{i}+k_{i N}+k_{i E}
$$

QUADRO 2 - INDICADORES DA INTEGRACÃO ESPACIAL

\begin{tabular}{|c|c|c|c|}
\hline $\begin{array}{c}\text { Destino } \\
\text { Origem }\end{array}$ & Região 1 & Região 2 & Região 3 \\
\hline Região 1 & $\mathrm{b}_{1}$ & $\mathrm{~m}_{12}$ & $\mathrm{~m}_{13}$ \\
\hline Região 2 & $\mathrm{m}_{21}$ & $\mathrm{~b}_{2}$ & $\mathrm{~m}_{23}$ \\
\hline Região 3 & $\mathrm{m}_{31}$ & $\mathrm{~m}_{32}$ & $\mathrm{~b}_{3}$ \\
\hline
\end{tabular}

\begin{tabular}{|c|c|c|c|}
\hline$X_{i N} / Y_{i}$ & $X_{i E} / Y_{i}$ & $X_{i} N_{i}$ & $Y_{i} / Y_{i}$ \\
\hline$k_{1 N}$ & $k_{1 E}$ & $k_{1}$ & 1 \\
\hline$k_{2 N}$ & $k_{2 E}$ & $k_{2}$ & 1 \\
\hline$k_{3 N}$ & $k_{3 E}$ & $k_{3}$ & 1 \\
\hline
\end{tabular}

\begin{tabular}{|l|c|c|c|}
\hline$M_{\mathrm{N} i} / Y_{i}$ & $m_{\mathrm{N} 1}$ & $m_{\mathrm{N} 2}$ & $m_{\mathrm{N} 3}$ \\
\hline$M_{\mathrm{E} i} Y_{i}$ & $m_{\mathrm{E} 1}$ & $m_{\mathrm{E} 2}$ & $m_{\mathrm{E} 3}$ \\
\hline$M_{\mathrm{i}} / Y_{i}$ & $m_{1}$ & $m_{2}$ & $m_{3}$ \\
\hline$D_{i} / Y_{i}$ & $d_{1}$ & $d_{2}$ & $d_{3}$ \\
\hline$\left(X_{i}-M_{i}\right) / Y_{i}$ & $s_{1}$ & $s_{2}$ & $s_{3}$ \\
\hline$Y_{i} / Y_{i}$ & $1_{1}$ & $1_{2}$ & $1_{3}$ \\
\hline
\end{tabular}

A relação (5) indica que cada unidade do produto regional se distribui para os mercados local, nacional e internacional, sendo $b_{i}\left(o u m_{i j}\right.$, para $\left.i=j\right)$ a propensão média da região a absorver o seu próprio produto, $k_{\mathrm{iN}}$ o coeficiente de abertura da região às demais regiões do país e $\mathrm{k}_{\mathrm{iE}} \mathrm{o}$ coeficiente de abertura da região ao exterior. A soma desses dois últimos coeficientes fornece o coeficiente de abertura da economia local, igual a $k_{i}$.

Dividindo-se as relações (2), (3) e (4) por $Y_{j}$, obtém-se os coeficientes da mobilidade inter-regional de bens e serviços, visualizados nas primeiras colunas do Quadro 2, como segue:

$$
\begin{aligned}
& 1=d_{j}+s_{j} \\
& d_{j}=b_{j}+m_{N j}+m_{E j} \quad e \\
& 1=m_{i j}+m_{2 j}+m_{3 j}+\ldots+m_{n j}+m_{E j}+s_{j}
\end{aligned}
$$

A relação (6) estabelece que cada unidade do produto regional é gasto integralmente ou poupado, em parte; sendo $\mathrm{d}_{\mathrm{j}}$ a propensão média a gastar e $\mathrm{s}_{\mathrm{j}} \mathrm{o}$ saldo médio da balança comercial $\mathrm{Na}$ relação (7), a propensão a gastar $\mathrm{d}_{\mathrm{j}}$ desdobra-se em propensão a absorver o próprio produto, $b_{j}$ e em propensão a importar do país $\left(m_{N j}\right)$ e do exterior $\left(m_{E j}\right)$. A equação $(8)$ fornece as propensões a importar de cada uma das regiões do sistema nacional, $m_{1 j}+m_{2 j}+\ldots+m_{n j} e a$ propensão a importar do exterior $\left(m_{\mathrm{Ej}}\right)$, além do saldo médio da balança comercial 
$\left(s_{j}\right)$ Quanto mais significativos forem os coeficientes $m_{i j}$, tanto mais as regiões $i$ e $j$ apresentam-se integradas.

\section{3. ÍNDICES DE ENCADEAMENTO E REGIÕES-CHAVE}

$O$ aumento da integração interindustrial de cada região $j$ engendra a expansằo do produto regional, pela ampliação do circuito local de difusão dos efeitos de encadeamento da produção e do emprego. As importações inter-regionais e internacionais, sendo função da renda de cada região, implicam que o aumento inicial do produto de uma região i, devido, por exemplo, à expansão da demanda externa por seus produtos, ou às mudanças tecnológicas internas, redutoras de custo, incrementa o comércio inter-regional e o produto de todas as regiões.

No contexto de uma economia aberta, portanto, os mesmos efeitos de encadeamento, existentes entre os setores de uma mesma região, também se verificam entre as diferentes economias regionais. Esses efeitos de encadeamento entre as atividades ligadas tecnologicamente dependem da distância, da tecnologia dos meios de transportes e dos custos de mobilidade espacial de bens, serviços e informações. Considerando-se o vetor das exportações internacionais $(X)$ como um vetor de variáveis exógenas, pode-se calcular os impactos diretos e indiretos sobre o vetor da produção inter-regional $(Y)$, ou encadeamentos verticais, como segue:

$$
Y=(I-B)^{-1} X \text {, sendo } B=M Y_{d}^{-1} \text { e } R=(1-B)^{-1}
$$

onde I é a matriz identidade; $B$ é a matriz dos coeficientes verticais diretos do comércio inter-regional; $M$ é a matriz dos fluxos inter-regionais de comércio e $Y_{d}^{-1}$ é a matriz diagonal inversa do produto de cada região. $R$ é a matriz dos impactos diretos e indiretos das exportações internacionais

$$
\delta \mathrm{Y}_{\mathrm{i}} / \delta \mathrm{X}_{\mathrm{j}}=\mathrm{rij}_{\mathrm{ij}}
$$

Cada elemento $r_{i j}$ de $\mathrm{R}$ representa o quanto da produção da região $i$ irá variar, direta $\mathrm{e}$ indiretamente, quando as exportações internacionais da região $j$ crescerem de uma unidade. Esse elemento indica também a demanda da região $j$ à região $i$ por conta dessa variação unitária das exportações internacionais da região $j$ (impacto sobre a demanda). Os impactos totais sobre a demanda na economia, provocados pela expansão unitária das exportações da região $i$, são dados por.

$$
r_{\cdot j}=\sum_{j=1}^{n} r_{i j}=r_{1 j}+r_{2 j}+\ldots+r_{n j}
$$

Onde $\sum_{i=1}^{n}$ indica a soma da coluna $j$, com $i$ variando de 1 até $\mathrm{n}$, sendo $\mathrm{n}$ o número de regiões do sistema nacional A estimativa do acréscimo direto e indireto do produto a ser ofertado por uma região $i$ escolhida aleatoriamente, quando as exportações da região $j$ aumentarem de uma unidade, é dada por:

$r_{j} / n$

Analogamente, os encadeamentos horizontais são obtidos de:

$$
Y^{\top}=V^{\top}(I-C)^{-1} \text {, sendo } C=Y_{d}^{-1} M e Z=(I-C)^{-1}
$$

onde $T$ indica transposição, $V$ é o vetor das importações internacionais; $C$ é a matriz dos coeficientes horizontais diretos do comércio inter-regional e $Z$ é a matriz dos impactos diretos e indiretos das importações internacionais 
Supõe-se que sejam constantes tanto os coeficientes de compras (tecnologia invariável), como os de vendas (market-share). A metodologia introduzida em (13) baseia-se na análise de Jones (1976) para os encadeamentos para a frente.

$\delta \gamma_{j} / \delta \mathrm{v}_{\mathrm{i}}=\mathrm{z}_{\mathrm{ij}}$

$\mathrm{O}$ elemento $z_{\mathrm{ij}}$ de $z$ representa o aumento direto e indireto da produção da região $j$, necessário para absorver o aumento da produção $i$, devido à expansão unitária de suas importações internacionais.

O aumento total da produção da economia requerido para absorver a variação unitária das importações internacionais da região $i$, é dado por

$$
z_{i}=\sum_{j=1}^{n} z_{i j}=z_{i 1}+z_{i 2}+\ldots+z_{i n}
$$

A estimativa do acréscimo direto e indireto do produto a ser demandado por uma região $j$ escolhida aleatoriamente, quando as importações internacionais de uma região $i$ aumentar de uma unidade, é dado por:

$z_{i} / n$

As estimativas médias (12) e (16) precisam ser normalizadas para possibilitar comparações com estimativas para regiões diferentes. Um critério é efetuar a normalização pela média global da soma dos coeficientes diretos e indiretos de comércio, como segue (Souza, 1988, p. 105)

$$
\left(1 / n^{2}\right)=\sum_{j=1}^{n} \sum_{i=1}^{n} r_{i j}=\left(1 / n^{2}\right) \sum_{j=1}^{n} r_{j} \quad \text { e } \quad\left(1 / n^{2}\right)=\sum_{i=1}^{n} \sum_{j=1}^{n} z_{i j}=1 / n^{2} \sum_{i=1}^{n} z_{i}
$$

Obtém-se, finalmente, 0 índice de encadeamento vertical das exportações internacionais, como segue:

$$
U_{j}=(1 / n) r_{j} /\left(1 / n^{2}\right) \sum_{j=1}^{n} r_{j}
$$

$O$ indice $U_{j}$ indica o encadeamento vertical médio normalizado de uma região $j$ sobre a oferta do conjunto de regiões, quando as exportações internacionais dessa região aumentarem de uma unidade $\mathrm{O}$ índice de encadeamento para a frente das importações internacionais $\left(U_{\mathrm{i}}\right)$ são obtidos de modo semelhante:

$$
U_{i}=(1 / n) z_{i} /\left(1 / n^{2}\right) \sum_{i=1}^{n} z_{i}
$$

$O$ índice $U_{i}$ fornece o encadeamento horizontal médio normalizado do produto a ser demandado pela economia, resultante de uma variação unitária das importações internacionais da região $i$.

Se $U_{j}>1$, a região exerce um impacto sobre a oferta da economia superior à média global do resto do sistema, quando suas exportações internacionais aumentarem de uma unidade. A região $j$ compra no mercado interno acima da média global da economia, exercendo, portanto, importantes efeitos de encadeamento para trás

Se $U_{i}>1$, a região $i$ coloca produtos a disposição da economia acima da média global, por conta do acréscimo de uma unidade de suas importações internacionais.

Uma região é considerada chave quando tanto o indice vertical como o índice horizontal são maiores que a unidade. A regiäo exerce, simultaneamente, efeitos 
acima da média global do sistema sobre a oferta e a demanda do conjunto das regiōes.

Podem ser calculados os coeficientes de variações dos índices $r_{i j}$ e $z_{i j}$, para excluir regiões-chave com efeitos de encadeamento muito concentrados (altos coeficientes de variação). Nas análises setoriais, os coeficientes de variação têm sido relativamente baixos, indicando que os inter-relacionamentos apresentam-se uniformemente distribuídos (Souza, 1995).

Os índices de encadeamento verticais e horizontais, referindo-se a variações unitárias das exportações ou das importações, são índices potenciais Torna-se necessário considerar o peso de cada região na economia. ${ }^{1}$ De outra parte, existe um conflito entre gerar maior volume de produção e criar empregos. Indústriaschave no encadeamento da produção não geram muito emprego (Souza, 1993)

Essas duas críticas podem ser contornadas pela ponderação das matrizes $R$ e $Z$ pelo peso da região i na economia $\left(Y_{i} / Y\right)$ e pelo seu coeficiente de emprego $\left(E_{i} / Y_{i}\right)$ Por simplificação, resulta a ponderação $\left(E_{i} / Y=e_{i}\right)$, que irá gerar as matrizes $R^{*} e$ $Z^{*}$, como segue

$$
R^{*}=\operatorname{Re}_{d} \text { e } Z^{*}=e_{d} Z
$$

$O$ coeficiente $E_{i} / Y$ corresponde ao peso de cada região i na economia do ponto de vista do emprego. A partir das matrizes $R^{*}$ e $Z^{*}$, calculam-se os índices de encadeamentos inter-regionais modificados, $U^{*}{ }_{j} e U^{*}$, que indicam tanto os efeitos reais totais sobre a produção, como sobre o emprego Uma região será considerada chave na geração da produção e do emprego quando os índices modificados $U^{\star}$; $e$ $\mathrm{U}_{\mathrm{i}}^{*}$ forem superiores à unidade.

\section{CONCLUSÃO}

O desenvolvimento dos meios de transporte entre as regiões favorece a mobilidade espacial dos bens, fatores e inovações tecnológicas A redução dos custos dessa mobilidade amplia as áreas de mercado e gera economias de escala para as firmas competitivas em qualquer ponto do espaço.

Dada a infra-estrutura de transportes e determinada distribuição espacial da atividade econômica, o crescimento de uma indústria em uma região específica exerce efeitos de encadeamento sobre o conjunto das regiões. Esses efeitos são mais intensos em alguns pontos do que em outros. Em geral, eles se diluem com o aumento da distância do local onde foram gerados. Em um momento do tempo, portanto, existe uma estrutura de fluxos inter-regionais de bens e serviços indicando o grau de integração entre as diferentes regiões de uma economia

Uma região será tanto mais integrada a uma outra quanto mais sua propensão a importar dessa região for elevada. A integração pode ser vista tanto pelo lado da compra de bens e serviços (integração vertical), como pelo lado das vendas (integração horizontal). Tendo em vista essa integração, uma região $j$ exerce efeitos de encadeamentos sobre as demais, quando a demanda internacional por suas exportações se eleva, ou quando realiza inovações tecnológicas (novos produtos ou novos processos redutores de custos). Isso ocorrendo, essa região j aumentará sua demanda de bens e serviços provenientes da própria área, das demais regiões do sistema nacional ou do exterior

\footnotetext{
${ }^{1}$ Vale a mesma observação a análise setorial, quando todas as indústrias têm o mesmo peso (Laumas, 1976)
} 
Por outro lado, ao aumentar suas exportações internacionais, seja para consumo intermediário ou final, uma região $i$ também exerce efeitos de encadeamento no conjunto da economia Sua produção aumenta, gerando impactos sobre as demais regiões, ao expandir a oferta de insumos e de produtos finais de consumo e ao demandar bens e serviços, tendo em vista as interdependências tecnológicas e o grau de integração comercial. Algumas regiões geram maiores impactos no espaço do que as outras. Aquelas que exercem, simultaneamente, efeitos de encadeamentos verticais e horizontais acima da média do sistema são regiõeschave na geração da produção e/ou do emprego.

Investimentos realizados nessas regiões provocam maiores impactos no resto do sistema, maximizando o crescimento da produção e do emprego. Obviamente que determinados investimentos em regiões mais pobres, com menores de encadeamentos, mesmo não gerando grandes impactos no sistema no curto prazo, criam condições mais favoráveis para maior crescimento no longo prazo, ao alterar a dotação de fatores, a estrutura inter-regional e a integração espacial.

No longo prazo, a integração econômica interna de cada região e o desenvolvimento dos canais para difusão espacial dos efeitos de encadeamento modificam as propensões a importar de cada área, bem como seus coeficientes de abertura. Com desenvolvimento, a estrutura produtiva de cada região tende a ficar mais homogênea, reduzindo-se as desigualdades regionais (Souza, 1995).

Seguindo Perroux (1955), pode-se afirmar que a importância do enfoque da integração espacial está na difusão do crescimento econômico a partir da regiãochave em expansão, quando todas as regiões interligadas podem-se beneficiar Obviamente, as crises porventura existertes na região-chave se farão sentir de um ponto a outro do território Contudo, na medida em que cada região possuir uma estrutura integrada e diversificada do ponto de vista interno, as crises externas não se manifestarão internamente de uma maneira muito intensa, a não ser no caso de uma depressão generalizada.

O conhecimento das regiões-chave e das regiões com fortes encadeamentos verticais e horizontais da produção e do emprego torna-se muito importante para a formulação de políticas de desenvolvimento regional $O$ modelo apresentado mostra, em síntese, que o bloqueio do crescimento de uma região entrava o desenvolvimento das demais e que o dinamismo de uma determinada área expande a economia como um todo. 


\section{BIBLIOGRAFIA}

BALASSA, Bela. The theory of economic integration New York Irwin, 1961

BERNIS, G. D Industries industrialisantes et contenu d'une politique d'intégration économique Economie Apliquée, n. 3/4, 1966

ERBES, Robert Intégration économique internationale Paris. P.UF , 1966.

JONES, Leroy P. The measurement of Hirshmanian linkages Quartely Journal of Economics, v $90, n$,,$p$ 323-333, May, 1976

LAUMAS, Prem $S$ The weighting problem in testing the linkage hypotesis. Quaterly Journal of Economics, $\vee 90$, n 2, p. 308-312, May, 1976

LEONTIEF, Wassaly input-output economics. New York: Oxford University Press, 1966

METZLER, L A multiple-region theory of income and trade Econometrica, Oct, p. 329-354, 1950

PERROUX, François Note sur la notion de pôle de croissance Économie Apliquée, n 17, p 307-320, 1955

SANSON, Joäo R. SOUZA, Nali J. Produtos-chave no encadeamento de preços In. SOUZA, N J (Org) A economia da Inflação. Porto Alegre. Editora da Universidade Federal do Rio Grande do Sul, 1992.

SOUZA, Nali de Jesus L'analyse de la croissance régionale par la théorie de la base économique. les cas du Rio Grande do Sul Paris: Université de Paris I (Panthéon-Sorbonne) Mémoire pour le DE A, 1976, $152 p$.

Mobilité des biens et intégration spatiale Paris: Université de Paris 1 (Panthéon-Sorbonne). Dossier de Recherche, 1977, 156p

Intégration économique régionale l'exemple des régions brésiliennes Thèse (dout. $3^{\mathrm{e}}$ cycle econ)

Université de Paris I (Panthéon-Sorbonne), 1978, 350p Conceito e aplicação da teoria da base econômica Perspectiva Econômica Säo Leopoldo. UNISINOS, v 10, n.25, p. 117-130, mar, 1980 O papel da agricultura na integraçâo intersetorial brasileira. São Paulo. Faculdade de Economia e

Administração $321 \mathrm{p}$. Tese (dout econ) IPE/USP, 1988 Desenvolvimento Económico São Paulo: Atlas, 1995

Desenvolvimento Polarizado e desequilibrios regionais no Brasil Porto Alegre. Análise Econômica,

ano 11, n. 19, p. $29-5.9,1993$

\section{ABSTRACT}

\section{KEY-REGIONS IN THE REGIONAL ECONOMIC INTEGRATION}

Considering a matrix of inter-regional commercial relations, this article establishes a model of spatial integration among different regions. The model provides some relations of spatial integration, mensures the inter-regionals linkage effects and defines the key-regions related to the growth in the space It also allows to maximize the spatial integration in the interior of the same country or in the context of integration areas such as Mercosul and NAFTA 